\title{
Random attractors for stochastic discrete complex non-autonomous Ginzburg-Landau equations with multiplicative noise
}

\author{
Peng Wang ${ }^{1}$, Yumei Huang ${ }^{2}$ and Xiaohu Wang ${ }^{3 *}$
}

\author{
"Correspondence: \\ wangxiaohu@scu.edu.cn \\ ${ }^{3}$ Colloge of Mathematics, Sichuan \\ University, Chengdu, 610064, China \\ Full list of author information is \\ available at the end of the article
}

\begin{abstract}
In this paper, we study the asymptotic behavior of stochastic discrete complex non-autonomous Ginzburg-Landau equations with multiplicative noise. We prove the existence and uniqueness of the random attractor.
\end{abstract}

MSC: 37L55; 37L30; 34F05

Keywords: random attractor; stochastic complex non-autonomous Ginzburg-Landau equation; multiplicative noise

\section{Introduction}

Recently, spatially discrete systems have drawn considerable attention, especially in the study of biological systems, atomic chains, solid state physics, electrical lattices, and BoseEinstein condensates. The different dynamical behavior of spatially discrete systems has been studied in many works, such as [1-3] for traveling waves solutions, [4-6] for chaos behavior and [7-13] for global attractors. In particularly, the discrete complex GinzburgLandau equation is encountered in several diverse branches of physics, ranging from superconductivity and nonlinear optics, to Bose-Einstein condensates. One of the most interesting applications is the description of the dynamical behavior of discrete complex Ginzburg-Landau equations. The existence of attractors for discrete complex GinzburgLandau equations is considered in $[14,15]$.

However, scientific and engineering systems are often subject to uncertainty or random influence. Therefore, it is significant and of prime importance to introduce random effects in the models. These random effects are not only introduced to compensate for the defects in some deterministic models, but also are often rather intrinsic phenomena. For example [16] has studied the Bose-Einstein condensation far from thermal equilibrium by solving the complex Ginzburg-Landau equation with a stochastic term. The existence of global random attractor was extensively studied, in [17-22] for systems with additive or multiplicative white noises on infinite lattice, in [23, 24] for systems with non-Gaussian noises on infinite lattice and especially in [25] for stochastic discrete Ginzburg-Landau equations with additive white noise. Note that these works only deal with autonomous systems which do not contain deterministic non-autonomous terms. When a stochastic equation does not contain deterministic non-autonomous terms, one can associate a ran-

(c) 2015 Wang et al. This article is distributed under the terms of the Creative Commons Attribution 4.0 International License (http://creativecommons.org/licenses/by/4.0/), which permits unrestricted use, distribution, and reproduction in any medium, provided you give appropriate credit to the original author(s) and the source, provide a link to the Creative Commons license, and indicate if changes were made. 
dom dynamical system with the equation over a probability space and then discuss the existence of random attractors of the system. The probability space can be considered as a parametric space. However, when dealing with stochastic equations with deterministic non-autonomous terms, we need two parametric spaces. One space is a nonempty set responsible for the deterministic non- autonomous terms; while the other space is a probability space responsible for the stochastic terms. The existence of random attractors for non-autonomous random dynamical systems was first proved in [26] and then in [27-30]. To the best of our knowledge, there are no results on the existence of random attractors for stochastic discrete complex non-autonomous Ginzburg-Landau equation with multiplicative noise. On the basis of this, this article is devoted to the discussion of this problem.

This paper is organized as follows. In Section 2, we introduce basic concepts concerning random dynamical systems and random attractors for non-autonomous random dynamical systems. In Section 3, we show the existence and uniqueness of the random attractor for stochastic discrete complex non-autonomous Ginzburg-Landau equation with multiplicative noise.

\section{Theory of random attractor}

In this section, we recall some results on random attractors for non-autonomous random dynamical systems with two parametric spaces from [26, 27]. This sort of dynamical systems can be generated by differential equations with both non-autonomous deterministic and stochastic external terms. All results given in this section are not original and they are presented here just for the reader's convenience. We also refer the reader to [31-33] for the theory of random attractors for autonomous random dynamical systems with one parametric space.

Let $(\Omega, \mathcal{F}, P)$ be a probability space and $\theta: \mathbb{R} \times \Omega \rightarrow \Omega$ be a $(\mathcal{B}(\mathbb{R}) \times \mathcal{F}, \mathcal{F})$-measurable mapping such that $\theta(0, \cdot)$ is the identity on $\Omega, \theta(s+t, \cdot)=\theta(t, \cdot) \circ \theta(s, \cdot)$ for all $t, s \in \mathbb{R}$ and $P \theta(t, \cdot)=P$ for all $t \in \mathbb{R}$. We usually write $\theta(t, \cdot)$ as $\theta_{t}$ and call $\left(\Omega, \mathcal{F}, P,\left\{\theta_{t}\right\}_{t \in \mathbb{R}}\right)$ a metric dynamical system.

Let $(X, d)$ be a complete separable metric space with Borel $\sigma$-algebra $\mathcal{B}(X)$. Given $r>0$ and $D \subseteq X$, the neighborhood of $D$ with radius $r$ is written as $\mathcal{N}_{r}(D)$. Denote by $2^{X}$ the collection of all subsets of $X$. A set-valued mapping $K: \mathbb{R} \times \Omega \rightarrow 2^{X}$ is called measurable with respect to $\mathcal{F}$ in $\Omega$ if the value $K(\tau, \omega)$ is a closed nonempty subset of $X$ for all $\tau \in \mathbb{R}$ and $\omega \in \Omega$, and the mapping $\omega \in \Omega \rightarrow d(x, K(\tau, \omega))$ is $(\mathcal{F}, \mathcal{B}(\mathbb{R}))$-measurable for every fixed $x \in X$ and $\tau \in \mathbb{R}$. If $K$ is measurable with respect to $\mathcal{F}$ in $\Omega$, then we say that the family $\{K(\tau, \omega): \tau \in \mathbb{R}, \omega \in \Omega\}$ is measurable with respect to $\mathcal{F}$ in $\Omega$. We now define a cocycle on $X$ over two parametric spaces.

Definition 2.1 Let $\left(\Omega, \mathcal{F}, P,\left\{\theta_{t}\right\}_{t \in \mathbb{R}}\right)$ be a metric dynamical system. A mapping $\Phi: \mathbb{R}^{+} \times$ $\mathbb{R} \times \Omega \times X \rightarrow X$ is called a continuous cocycle on $X$ over $\mathbb{R}$ and $\left(\Omega, \mathcal{F}, P,\left\{\theta_{t}\right\}_{t \in \mathbb{R}}\right)$ if for all $\tau \in \mathbb{R}, \omega \in \Omega$ and $t, s \in \mathbb{R}^{+}$, the following conditions (i)-(iv) are satisfied:

(i) $\Phi(\cdot, \tau, \cdot, \cdot): \mathbb{R}^{+} \times \Omega \times X \rightarrow X$ is $\left(\mathcal{B}\left(\mathbb{R}^{+}\right) \times \mathcal{F} \times \mathcal{B}(X), \mathcal{B}(X)\right)$-measurable;

(ii) $\Phi(0, \tau, \omega, \cdot)$ is the identity on $X$;

(iii) $\Phi(t+s, \tau, \omega, \cdot)=\Phi\left(t, \tau+s, \theta_{s} \omega, \cdot\right) \circ \Phi(s, \tau, \omega, \cdot)$;

(iv) $\Phi(t, \tau, \omega, \cdot): X \rightarrow X$ is continuous. 
If, in addition, there exists a positive number $T$ such that for every $t \in \mathbb{R}^{+}, \tau \in \mathbb{R}$ and $\omega \in \Omega$,

$$
\Phi(t, \tau+T, \omega, \cdot)=\Phi(t, \tau, \omega, \cdot)
$$

then $\Phi$ is called a continuous periodic cocycle on $X$ with period $T$.

Definition 2.2 A collection $\mathcal{D}$ of some families of nonempty subsets of $X$ is said to be neighborhood closed if for each $D=\{D(\tau, \omega): \tau \in \mathbb{R}, \omega \in \Omega\} \in \mathcal{D}$, there is a positive number $\epsilon$ depending on $D$ such that the family

$$
\left\{B(\tau, \omega): B(\tau, \omega) \text { is a nonempty subset of } \mathcal{N}_{\epsilon}(D(\tau, \omega)), \forall \tau \in \mathbb{R}, \omega \in \Omega\right\}
$$

also belongs to $\mathcal{D}$.

Hereafter, we assume $\Phi$ is a continuous cocycle on $X$ over $\mathbb{R}$ and $\left(\Omega, \mathcal{F}, P,\left\{\theta_{t}\right\}_{t \in \mathbb{R}}\right)$, and $\mathcal{D}$ is the collection of all tempered families of nonempty bounded subsets of $X$. Remember that a family $D=\{D(\tau, \omega): \tau \in \mathbb{R}, \omega \in \Omega\}$ of nonempty bounded subsets of $X$ is said to be tempered if for every $c>0$, the following holds:

$$
\lim _{t \rightarrow-\infty} e^{c t}\left\|D\left(\tau+t, \theta_{t} \omega\right)\right\|_{X}=0
$$

where $\|D\|_{X}=\sup _{x \in D}\|x\|_{X}$.

Definition 2.3 A mapping $\psi: \mathbb{R} \times \mathbb{R} \times \Omega \rightarrow X$ is called a complete orbit of $\Phi$ if for every $t \in \mathbb{R}^{+}, \tau, s \in \mathbb{R}$, and $\omega \in \Omega$, the following holds:

$$
\Phi\left(t, \tau+s, \theta_{s} \omega, \psi(s, \tau, \omega)\right)=\psi(t+s, \tau, \omega)
$$

If, in addition, there exists $D=\{D(\tau, \omega): \tau \in \mathbb{R}, \omega \in \Omega\} \in \mathcal{D}$ such that $\psi(t, \tau, \omega)$ belongs to $D\left(\tau+t, \theta_{t} \omega\right)$ for every $t \in \mathbb{R}, \tau \in \mathbb{R}$, and $\omega \in \Omega$, then $\psi$ is called a $\mathcal{D}$-complete orbit of $\Phi$.

Definition 2.4 Let $B=\{B(\tau, \omega): \tau \in \mathbb{R}, \omega \in \Omega\}$ be a family of nonempty subsets of $X$. For every $\tau \in \mathbb{R}$ and $\omega \in \Omega$, let

$$
\Omega(B, \tau, \omega)=\bigcap_{r \geq 0} \overline{\bigcup_{t \geq r} \Phi\left(t, \tau-t, \theta_{-t} \omega, B\left(\tau-t, \theta_{-t} \omega\right)\right)} .
$$

Then the family $\{\Omega(B, \tau, \omega): \tau \in \mathbb{R}, \omega \in \Omega\}$ is called the $\Omega$-limit set of $B$ and is denoted by $\Omega(B)$.

Definition 2.5 A family $K=\{K(\tau, \omega): \tau \in \mathbb{R}, \omega \in \Omega\} \in \mathcal{D}$ is called a $\mathcal{D}$-pullback absorbing set for $\Phi$ if for all $\tau \in \mathbb{R}, \omega \in \Omega$ and for every $D \in \mathcal{D}$, there exists $T=T(D, \tau, \omega)>0$ such that

$$
\Phi\left(t, \tau-t, \theta_{-t} \omega, D\left(\tau-t, \theta_{-t} \omega\right)\right) \subseteq K(\tau, \omega) \quad \text { for all } t \geq T \text {. }
$$


If, in addition, for all $\tau \in \mathbb{R}$ and $\omega \in \Omega, K(\tau, \omega)$ is a closed nonempty subset of $X$ and $K$ is measurable in $\omega$ with respect to $\mathcal{F}$ in $\Omega$, then we say $K$ is a closed measurable $\mathcal{D}$-pullback absorbing set for $\Phi$.

Definition 2.6 The cocycle $\Phi$ is said to be $\mathcal{D}$-pullback asymptotically compact in $X$ if for all $\tau \in \mathbb{R}$ and $\omega \in \Omega$, the sequence

$$
\left\{\Phi\left(t_{n}, \tau-t_{n}, \theta_{-t_{n}} \omega, x_{n}\right)\right\}_{n=1}^{\infty} \text { has a convergent subsequence in } X
$$

whenever $t_{n} \rightarrow \infty$, and $x_{n} \in B\left(\tau-t_{n}, \theta_{-t_{n}} \omega\right)$ with $\{B(\tau, \omega): \tau \in \mathbb{R}, \omega \in \Omega\} \in \mathcal{D}$.

Definition 2.7 A family $\mathcal{A}=\{\mathcal{A}(\tau, \omega): \tau \in \mathbb{R}, \omega \in \Omega\} \in \mathcal{D}$ is called a $\mathcal{D}$-pullback attractor for $\Phi$ if the following conditions (i)-(iii) are fulfilled:

(i) $\mathcal{A}$ is measurable in $\omega$ with respect to $\mathcal{F}$ in $\Omega$ and $\mathcal{A}(\tau, \omega)$ is compact in $X$ for all $\tau \in \mathbb{R}$ and $\omega \in \Omega$.

(ii) $\mathcal{A}$ is invariant, that is, for every $\tau \in \mathbb{R}$ and $\omega \in \Omega$,

$$
\Phi(t, \tau, \omega, \mathcal{A}(\tau, \omega))=\mathcal{A}\left(\tau+t, \theta_{t} \omega\right), \quad \forall t \geq 0
$$

(iii) $\mathcal{A}$ attracts every member of $\mathcal{D}$, that is, for every $D=\{D(\tau, \omega): \tau \in \mathbb{R}, \omega \in \Omega\} \in \mathcal{D}$ and for every $\tau \in \mathbb{R}$ and $\omega \in \Omega$,

$$
\lim _{t \rightarrow \infty} d\left(\Phi\left(t, \tau-t, \theta_{-t} \omega, D\left(\tau-t, \theta_{-t} \omega\right)\right), \mathcal{A}(\tau, \omega)\right)=0
$$

If, in addition, there exists $T>0$ such that

$$
\mathcal{A}(\tau+T, \omega)=\mathcal{A}(\tau, \omega), \quad \forall \tau \in \mathbb{R}, \forall \omega \in \Omega
$$

then we say $\mathcal{A}$ is periodic with period $T$.

The following result on the existence and uniqueness of $\mathcal{D}$-pullback attractors for continuous cocycle can be found in [26, 27]. Similar results can be found in [31-33] for autonomous random dynamical systems.

Proposition 2.1 Let $\mathcal{D}$ be a neighborhood closed collection of some families of nonempty subsets of $X$, and $\Phi$ be a continuous cocycle on $X$ over $\mathbb{R}$ and $\left(\Omega, \mathcal{F}, P,\left\{\theta_{t}\right\}_{t \in \mathbb{R}}\right)$. Then $\Phi$ has a $\mathcal{D}$-pullback attractor $\mathcal{A}$ in $\mathcal{D}$ if and only if $\Phi$ is $\mathcal{D}$-pullback asymptotically compact in $X$ and $\Phi$ has a closed measurable $\mathcal{D}$-pullback absorbing set $K$ in $\mathcal{D}$. The $\mathcal{D}$-pullback attractor $\mathcal{A}$ is unique and is given by for each $\tau \in \mathbb{R}$ and $\omega \in \Omega$,

$$
\begin{aligned}
\mathcal{A}(\tau, \omega) & =\Omega(K, \tau, \omega)=\bigcup_{D \in \mathcal{D}} \Omega(D, \tau, \omega) \\
& =\{\psi(0, \tau, \omega): \psi \text { is a } \mathcal{D} \text {-complete orbit of } \Phi\} .
\end{aligned}
$$

If, in addition, both $\Phi$ and $K$ are T-periodic, then so is the attractor $\mathcal{A}$. 


\section{Main results}

Denote by $\mathbb{Z}$ the set of integers. We denote by $\ell^{p}(p \geq 1)$ defined by

$$
\ell^{p}=\left\{u \mid u=\left(u_{n}\right)_{n \in \mathbb{Z}} \in \mathbb{C} \text {, and } \sum_{n \in \mathbb{Z}}\left|u_{n}\right|^{p}<+\infty\right\},
$$

with the norm $\|\cdot\|_{p}$ given by

$$
\|u\|_{p}=\left(\sum_{n \in \mathbb{Z}}\left|u_{n}\right|^{p}\right)^{1 / p}
$$

for any $u=\left(u_{n}\right)_{n \in \mathbb{Z}} \in \ell^{p}$.

In particular, $\ell^{2}$ is a Hilbert space with the inner product $(\cdot, \cdot)$ and norm $\|\cdot\|$ given by

$$
(u, v)=\sum_{n \in \mathbb{Z}} u_{n} \bar{v}_{n}, \quad\|u\|=\left(\sum_{n \in \mathbb{Z}}\left|u_{n}\right|^{2}\right)^{1 / 2},
$$

for any $u=\left(u_{n}\right)_{n \in \mathbb{Z}}$ and $v=\left(v_{n}\right)_{n \in \mathbb{Z}} \in \ell^{2}$, where $\bar{v}_{n}$ denotes the conjugate of $v_{n}$.

Let $\tau \in \mathbb{R}$. In this paper we consider the following discrete complex non-autonomous Ginzburg-Landau equation with multiplicative noise:

$$
\begin{aligned}
d u_{n}= & \left((\lambda+\mathrm{i} \alpha)\left(u_{n-1}-2 u_{n}+u_{n+1}\right)-(\gamma+\mathrm{i} \mu) u_{n}\right. \\
& \left.-(\kappa+\mathrm{i} \beta)\left|u_{n}\right|^{p} u_{n}+g_{n}(t)\right) d t+\sum_{m=1}^{N} c_{m} u_{n} \circ d \omega_{m}(t), \quad t>\tau,
\end{aligned}
$$

with the initial condition

$$
u_{n}(\tau)=u_{\tau, n}, \quad n \in \mathbb{Z}
$$

where $p>0, \lambda>0, \gamma>0, \kappa>0, \alpha, \beta, \mu \in \mathbb{R}$, $\mathrm{i}$ is the unit of imaginary numbers such that $\mathrm{i}^{2}=$ $-1, u=\left(u_{n}\right)_{n \in \mathbb{Z}}, g(t)=\left(g_{n}(t)\right)_{n \in \mathbb{Z}} \in L_{\text {loc }}^{2}\left(\mathbb{R}, \ell^{2}\right), c_{m} \in \mathbb{R}$ for $m=1, \ldots, N, w_{m}(t)$ are mutually independent Brownian motions on a probability space, and the symbol $\circ$ indicates that the equation is understood in the sense of Stratonovich integration.

Denote by $B, B^{*}, A$ and $C_{m}$ for $m=1, \ldots, N$ the linear operators from $\ell^{2}$ into $\ell^{2}$ in the following way: for any $u=\left(u_{n}\right)_{n \in \mathbb{Z}} \in \ell^{2}$,

$$
(B u)_{n}=u_{n+1}-u_{n}, \quad\left(B^{*} u\right)_{n}=u_{n-1}-u_{n}, \quad\left(C_{m} u\right)_{n}=c_{m} u_{n},
$$

and

$$
(A u)_{n}=2 u_{n}-u_{n-1}-u_{n+1}, \quad \text { for each } n \in \mathbb{Z} .
$$

Then we find that $A=B B^{*}=B^{*} B$ and $\left(B^{*} u, v\right)=(u, B v)$ for all $u, v \in \ell^{2}$. Therefore, $(A u, u) \geq$ 0 for all $u \in \ell^{2}$. 
The problem (3.1)-(3.2) can be regarded as follows: for $\tau \in \mathbb{R}$,

$$
\begin{aligned}
d u(t)= & (-(\lambda+\mathrm{i} \alpha) A u-(\gamma+\mathrm{i} \mu) u-(\kappa+\mathrm{i} \beta) f(u)+g(t)) d t \\
& +\sum_{m=1}^{N} C_{m} u \circ d w_{m}(t), \quad t>\tau,
\end{aligned}
$$

with the initial condition

$$
u(\tau)=u_{\tau},
$$

where $u=\left(u_{n}\right)_{n \in \mathbb{Z}}$, and the nonlinear term $f$ is defined as

$$
f(u(t))=|u(t)|^{p} u(t)=\left(\left|u_{n}(t)\right|^{p} u_{n}(t)\right)_{n \in \mathbb{Z}} .
$$

We now specify the probability space. Denote by

$$
\Omega=\{\omega \in C(\mathbb{R}, \mathbb{R}): \omega(0)=0\} .
$$

Let $\mathcal{F}$ be the Borel $\sigma$-algebra induced by the compact-open topology of $\Omega$, and $P$ the corresponding Wiener measure on $(\Omega, \mathcal{F})$. There is a classical group $\left\{\theta_{t}\right\}_{t \in \mathbb{R}}$ acting on $(\Omega, \mathcal{F}, P)$, which is defined by

$$
\theta_{t} \omega(\cdot)=\omega(\cdot+t)-\omega(t), \quad \omega \in \Omega, t \in \mathbb{R} .
$$

Then $\left(\Omega, \mathcal{F}, P,\left\{\theta_{t}\right\}_{t \in \mathbb{R}}\right)$ is a metric dynamical system (see [34]).

On the other hand, let us consider the one-dimensional stochastic differential equation

$$
d z+\alpha z d t=d w(t)
$$

for $\alpha>0$. This equation has a random fixed point in the sense of random dynamical systems generating a stationary solution known as the stationary Ornstein-Uhlenbeck process (see $[35,36]$ for more details). In fact, we have the following.

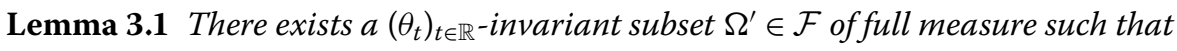

$$
\lim _{t \rightarrow \pm \infty} \frac{|\omega(t)|}{t}=0 \quad \text { for all } \omega \in \Omega^{\prime}
$$

and, for such $\omega$, the random variable given by

$$
z^{*}(\omega)=-\alpha \int_{-\infty}^{0} e^{\alpha s} \omega(s) d s
$$

is well defined. Moreover, for $\omega \in \Omega^{\prime}$, the mapping

$$
(t, \omega) \rightarrow z^{*}\left(\theta_{t} \omega\right)=-\alpha \int_{-\infty}^{0} e^{\alpha s} \theta_{t} \omega(s) d s=-\alpha \int_{-\infty}^{0} e^{\alpha s} \omega(t+s) d s+\omega(t)
$$


is a stationary solution of (3.6) with continuous trajectories. In addition, for $\omega \in \Omega^{\prime}$,

$$
\begin{aligned}
& \lim _{t \rightarrow \pm \infty} \frac{\left|z^{*}\left(\theta_{t} \omega\right)\right|}{|t|}=0, \quad \lim _{t \rightarrow \pm \infty} \frac{1}{t} \int_{0}^{t} z^{*}\left(\theta_{s} \omega\right) d s=0, \\
& \lim _{t \rightarrow \pm \infty} \frac{1}{t} \int_{0}^{t}\left|z^{*}\left(\theta_{s} \omega\right)\right| d s=\mathbb{E}\left|z^{*}\right|<\infty .
\end{aligned}
$$

Remark 3.1 We now consider $\theta$ defined in (3.5) on $\Omega^{\prime}$ instead of $\Omega$. This mapping possesses the same properties as the original one if we choose for $\mathcal{F}$ the trace $\sigma$-algebra with respect to $\Omega^{\prime}$ denoted also by $\mathcal{F}$.

Denote by $z_{m}^{*}$ the associated Ornstein-Uhlenbeck process corresponding to (3.6) with $\alpha=1$ and $w$ replaced by $w_{m}$ for $m=1, \ldots, N$. Then, for any $m=1, \ldots, N$, we have a stationary Ornstein-Uhlenbeck process generated by a random variable $z_{m}^{*}(\omega)$ on $\Omega_{m}^{\prime}$ with properties formulated in Lemma 3.1 defined on the metric dynamical system $\left(\Omega_{m}^{\prime}, \mathcal{F}_{m}, P_{m}, \theta\right)$. We set $(\tilde{\Omega}, \mathcal{F}, P, \theta)$, where

$$
\tilde{\Omega}=\Omega_{1}^{\prime} \times \cdots \times \Omega_{N}^{\prime}, \quad \mathcal{F}=\bigotimes_{m=1}^{N} \mathcal{F}_{m}, \quad P=P_{1} \times \cdots \times P_{N},
$$

and $\theta$ is the flow of the Wiener shifts.

Now, let us note that the operator $C_{m}$ generates a strongly continuous semigroup (in fact, a uniformly continuous group) of operators $S_{C_{m}}(t)$. More precisely, $S_{C_{m}}(t)$ is given by

$$
S_{C_{m}}(t) u=e^{c_{m} t} u, \quad \text { for } u \in \ell^{2} \text {. }
$$

Then we denote for every $\omega \in \tilde{\Omega}$,

$$
T(\omega):=S_{C_{1}}\left(z_{1}^{*}(\omega)\right) \circ \cdots \circ S_{C_{N}}\left(z_{N}^{*}(\omega)\right)=e^{\sum_{m=1}^{N} c_{m} z_{m}^{*}(\omega)} \operatorname{Id}_{\ell^{2}}
$$

which is clearly a homeomorphism in $\ell^{2}$. The inverse operator is well defined by

$$
T^{-1}(\omega):=S_{C_{N}}\left(-z_{N}^{*}(\omega)\right) \circ \cdots \circ S_{C_{1}}\left(-z_{1}^{*}(\omega)\right)=e^{-\sum_{m=1}^{N} c_{m} z_{m}^{*}(\omega)} \operatorname{Id}_{\ell^{2}}
$$

For simplicity, let us denote

$$
\delta(\omega)=\sum_{m=1}^{N} c_{m} z_{m}^{*}(\omega)
$$

It easily follows that $\left\|T^{-1}\left(\theta_{t} \omega\right)\right\|$ has sub-exponential growth as $t \rightarrow \pm \infty$ for any $\omega \in \tilde{\Omega}$. Hence $\left\|T^{-1}\right\|$ is tempered. According to Remark 3.1, we can change our metric dynamical system with respect to $\Omega^{\prime}$. However, we use the old notation $(\Omega, \mathcal{F}, P, \theta)$ to denote the new metric dynamical system.

For our purpose, we need to convert the stochastic equation (3.3)-(3.4) with a random term into a deterministic one with a random parameter. To this end, let us consider the change in variable

$$
v(t)=T^{-1}\left(\theta_{t} \omega\right) u(t)=e^{-\delta\left(\theta_{t} \omega\right)} u(t)
$$


where $u(t)$ is the solution of (3.3)-(3.4). Then we have for $\tau \in \mathbb{R}$,

$$
\begin{aligned}
d \nu(t)= & e^{-\delta\left(\theta_{t} \omega\right)} d u(t)-\sum_{m=1}^{N} c_{m} e^{-\delta\left(\theta_{t} \omega\right)} u(t) \circ d z_{m}^{*}\left(\theta_{t} \omega\right) \\
= & {\left[-(\lambda+\mathrm{i} \alpha) A v-(\gamma+\mathrm{i} \mu) v-(\kappa+\mathrm{i} \beta) e^{-\delta\left(\theta_{t} \omega\right)} f\left(e^{\delta\left(\theta_{t} \omega\right)} v\right)\right.} \\
& \left.+e^{-\delta\left(\theta_{t} \omega\right)} g(t)+\delta\left(\theta_{t} \omega\right) v(t)\right] d t, \quad t>\tau,
\end{aligned}
$$

with the initial value condition

$$
v(\tau)=e^{-\delta\left(\theta_{\tau} \omega\right)} u_{\tau} \triangleq v_{\tau}
$$

Therefore, by the standard method [37], we find that, for every $\tau \in \mathbb{R}, \omega \in \Omega$ and $v_{\tau} \in$ $\ell^{2}$, problem (3.9)-(3.10) has a unique global solution in $\ell^{2}$. In addition, this solution is measurable in $\omega \in \Omega$, and depends continuously on the initial data $v_{\tau}$. Consequently, we can associate a cocycle for problem (3.3)-(3.4) in $\ell^{2}$. Let $\Phi: \mathbb{R}^{+} \times \mathbb{R} \times \Omega \times \ell^{2} \rightarrow \ell^{2}$ be defined by

$$
\Phi\left(t, \tau, \omega, u_{\tau}\right)=u\left(t+\tau, \tau, \theta_{-\tau} \omega, u_{\tau}\right)=e^{\delta\left(\theta_{t} \omega\right)} v\left(t+\tau, \tau, \theta_{-\tau} \omega, v_{\tau}\right)
$$

where $v\left(t+\tau, \tau, \theta_{-\tau} \omega, v_{\tau}\right)$ is the solution of problem (3.9)-(3.10). Then $\Phi$ satisfies conditions (i)-(iv) in Definition 2.1. Therefore, it is a continuous cocycle associated with problem (3.3)-(3.4).

In the sequel, we will study tempered pullback attractors of $\Phi$ in $\ell^{2}$. As usual, we use $\mathcal{D}$ to denote the collection of all tempered families of nonempty bounded subsets of $\ell^{2}$. By definition, a family $D=\{D(\tau, \omega): \tau \in \mathbb{R}, \omega \in \Omega\}$ of nonempty bounded subsets of $\ell^{2}$ is tempered if for every $c>0$,

$$
\lim _{t \rightarrow-\infty} e^{c t}\left\|D\left(\tau+t, \theta_{t} \omega\right)\right\|=0
$$

where $\|D\|=\sup _{\psi \in D}\|\psi\|$.

Now, we establish uniform estimates of solutions and then derive uniform estimates on the tails of solutions of system (3.9)-(3.10). These estimates will be used to prove the existence of tempered random attractors. The following condition will be needed for $g$ when deriving uniform estimates of solutions:

$$
\int_{-\infty}^{0} e^{\frac{\gamma}{2} s}\|g(s+\tau)\|^{2} d s<\infty, \quad \forall \tau \in \mathbb{R}
$$

When constructing tempered pullback attractors, we will assume that

$$
\lim _{t \rightarrow-\infty} e^{c t} \int_{-\infty}^{0} e^{\frac{\gamma}{2} s}\|g(s+t)\|^{2} d s=0, \quad \forall c>0
$$

Lemma 3.2 Suppose (3.12) holds. Then for every $\tau \in \mathbb{R}, \omega \in \Omega$, and $D=\{D(\tau, \omega): \tau \in$ $\mathbb{R}, \omega \in \Omega\} \in \mathcal{D}$, there exists $T=T(\tau, \omega, D, \varepsilon)>0$ such that, for all $t \geq T$ and $v_{\tau-t} \in D(\tau-$ 
$\left.t, \theta_{-t} \omega\right)$, the solution $v$ of system (3.9)-(3.10) satisfies

$$
\begin{aligned}
& \left\|\nu\left(\tau, \tau-t, \theta_{-\tau} \omega, v_{\tau-t}\right)\right\|^{2}+\frac{\gamma}{2} \int_{-t}^{0} e^{\gamma s-2 \int_{0}^{s} \delta\left(\theta_{r} \omega\right) d r}\left\|v\left(s+\tau, \tau-t, \theta_{-\tau} \omega, \nu_{\tau-t}\right)\right\|^{2} d s \\
& \leq \frac{4}{\gamma} \int_{-\infty}^{0} e^{\gamma r+2 \int_{r}^{0} \delta\left(\theta_{s} \omega\right) d s} e^{-2 \delta\left(\theta_{r} \omega\right)}\|g(r+\tau)\|^{2} d r .
\end{aligned}
$$

Proof Taking the inner product of (3.9) with $v$ in $\ell^{2}$ and taking the real part, we get

$$
\begin{aligned}
\frac{d}{d t}\|v(t)\|^{2} & \leq-2 \gamma\|v\|^{2}+2\left(e^{-\delta\left(\theta_{t} \omega\right)} g(t), v\right)+2 \delta\left(\theta_{t} \omega\right)\|v\|^{2} \\
& \leq-\left(\frac{3}{2} \gamma-2 \delta\left(\theta_{t} \omega\right)\right)\|v\|^{2}+\frac{2}{\gamma} e^{-2 \delta\left(\theta_{t} \omega\right)}\|g(t)\|^{2} .
\end{aligned}
$$

Multiplying (3.15) by $e^{\gamma t-2 \int_{0}^{t} \delta\left(\theta_{s} \omega\right) d s}$ and then integrating over $(\tau-t, \tau)$ with $t \in \mathbb{R}^{+}$, we get, for every $\omega \in \Omega$,

$$
\begin{aligned}
& \left\|v\left(\tau, \tau-t, \omega, v_{\tau-t}\right)\right\|^{2}+\frac{\gamma}{2} \int_{\tau-t}^{\tau} e^{\gamma(s-\tau)-2 \int_{\tau}^{s} \delta\left(\theta_{r} \omega\right) d r}\left\|v\left(s, \tau-t, \omega, v_{\tau-t}\right)\right\|^{2} d s \\
& \leq e^{-\gamma t+2 \int_{\tau-t}^{\tau} \delta\left(\theta_{s} \omega\right) d s}\left\|v_{\tau-t}\right\|^{2}+\frac{2}{\gamma} \int_{\tau-t}^{\tau} e^{\gamma(r-\tau)+2 \int_{r}^{\tau} \delta\left(\theta_{s} \omega\right) d s} e^{-2 \delta\left(\theta_{r} \omega\right)}\|g(r)\|^{2} d r .
\end{aligned}
$$

Replacing $\omega$ by $\theta_{-\tau} \omega$ in (3.16), we obtain, for every $t \in \mathbb{R}^{+}, \tau \in \mathbb{R}$ and $\omega \in \Omega$,

$$
\begin{gathered}
\left\|\nu\left(\tau, \tau-t, \theta_{-\tau} \omega, v_{\tau-t}\right)\right\|^{2}+\frac{\gamma}{2} \int_{-t}^{0} e^{\gamma s-2 \int_{0}^{s} \delta\left(\theta_{r} \omega\right) d r}\left\|v\left(s+\tau, \tau-t, \theta_{-\tau} \omega, \nu_{\tau-t}\right)\right\|^{2} d s \\
\leq e^{-\gamma t+2 \int_{-t}^{0} \delta\left(\theta_{s} \omega\right) d s}\left\|v_{\tau-t}\right\|^{2}+\frac{2}{\gamma} \int_{-t}^{0} e^{\gamma r+2 \int_{r}^{0} \delta\left(\theta_{s} \omega\right) d s} e^{-2 \delta\left(\theta_{r} \omega\right)}\|g(r+\tau)\|^{2} d r .
\end{gathered}
$$

Because of the properties of the Ornstein-Uhlenbeck process $z_{m}^{*}$, there exists $T_{1}=T_{1}(\omega)>$ 0 such that $\int_{-t}^{0} \delta\left(\theta_{s} \omega\right) d s \leq \frac{\gamma}{4} t$ for all $t \geq T_{1}$. Since $v_{\tau-t} \in D\left(\tau-t, \theta_{-t} \omega\right)$ and $D$ is tempered, we find hat

$$
\limsup _{t \rightarrow \infty} e^{-\gamma t+2 \int_{-t}^{0} \delta\left(\theta_{s} \omega\right) d s}\left\|v_{\tau-t}\right\|^{2} \leq \limsup _{t \rightarrow \infty} e^{-\frac{\gamma}{2} t}\left\|D\left(\tau-t, \theta_{-t} \omega\right)\right\|^{2}=0 .
$$

By (3.12) and the properties of $z_{m}^{*}$ again, we see the following integral is convergent:

$$
\int_{-\infty}^{0} e^{\gamma r+2 \int_{r}^{0} \delta\left(\theta_{s} \omega\right) d s} e^{-2 \delta\left(\theta_{r} \omega\right)}\|g(r+\tau)\|^{2} d r<\infty
$$

It follows from (3.18) and (3.19) that there exists $T=T(\tau, \omega, D)>0$ such that, for all $t \geq T$,

$$
\begin{aligned}
& \left\|\nu\left(\tau, \tau-t, \theta_{-\tau} \omega, v_{\tau-t}\right)\right\|^{2}+\frac{\gamma}{2} \int_{-t}^{0} e^{\gamma s-2 \int_{0}^{s} \delta\left(\theta_{r} \omega\right) d r}\left\|v\left(s+\tau, \tau-t, \theta_{-\tau} \omega, \nu_{\tau-t}\right)\right\|^{2} d s \\
& \leq \frac{4}{\gamma} \int_{-\infty}^{0} e^{\gamma r+2 \int_{r}^{0} \delta\left(\theta_{s} \omega\right) d s} e^{-2 \delta\left(\theta_{r} \omega\right)}\|g(r+\tau)\|^{2} d r,
\end{aligned}
$$

which implies the desired estimates. 
Lemma 3.3 Suppose that (3.12) holds. Then for every $\tau \in \mathbb{R}, \omega \in \Omega, D=\{D(\tau, \omega): \tau \in$ $\mathbb{R}, \omega \in \Omega\} \in \mathcal{D}$ and $\varepsilon>0$, there exist $T=T(\tau, \omega, D, \varepsilon)>0$ and $\hat{N}=\hat{N}(\tau, \omega, \varepsilon)>0$ such that, for all $t \geq T$ and $v_{\tau-t} \in D\left(\tau-t, \theta_{-t} \omega\right)$, the solution of problem (3.1)-(3.2) satisfies

$$
\sum_{|n| \geq \hat{N}}\left|v_{n}\left(\tau, \tau-t, \theta_{-\tau} \omega, v_{\tau-t}\right)\right|^{2} \leq \varepsilon
$$

Proof Let $\rho(\cdot)$ be a smooth function defined on $\mathbb{R}^{+}$such that $0 \leq \rho(s) \leq 1$ for all $s \in \mathbb{R}^{+}$, and

$$
\rho(s)= \begin{cases}0, & \text { for } 0 \leq s \leq 1 \\ 1, & \text { for } s \geq 2\end{cases}
$$

Then there exists a constant $C_{0}$ such that $\left|\rho^{\prime}(s)\right| \leq C_{0}$ for $s \in \mathbb{R}^{+}$.

Let $k$ be a fixed positive integer which will be specified later, and set $x=\left(x_{n}\right)_{n \in \mathbb{Z}}$ with $x_{n}=\rho\left(\frac{|n|}{k}\right) v_{n}$. Taking the inner product of (3.9) with $x$ in $\ell^{2}$ and taking the real part, we get

$$
\begin{aligned}
\frac{d}{d t} \sum_{j \in \mathbb{Z}} \rho\left(\frac{|n|}{k}\right)\left|v_{n}\right|^{2} \leq & -2 \operatorname{Re}((\lambda+\mathrm{i} \alpha) A v, x)-2\left(\gamma-\delta\left(\theta_{t} \omega\right)\right) \sum_{n \in \mathbb{Z}} \rho\left(\frac{|n|}{k}\right)\left|v_{n}\right|^{2} \\
& +2 e^{-\delta\left(\theta_{t} \omega\right)}(g(t), x) .
\end{aligned}
$$

We now estimate the terms in (3.23) as follows. First, we have

$$
\begin{aligned}
\operatorname{Re}((\lambda+\mathrm{i} \alpha) A v, x)= & \operatorname{Re}(\lambda+\mathrm{i} \alpha)(B v, B x) \\
= & \operatorname{Re}(\lambda+\mathrm{i} \alpha) \sum_{n \in \mathbb{Z}}\left(v_{n+1}-v_{n}\right)\left(\bar{x}_{n+1}-\bar{x}_{n}\right) \\
= & \lambda \sum_{n \in \mathbb{Z}}\left(\rho\left(\frac{|n+1|}{k}\right)\left|v_{n+1}\right|^{2}+\rho\left(\frac{|n|}{k}\right)\left|v_{n}\right|^{2}\right) \\
& +\alpha \operatorname{Im} \sum_{n \in \mathbb{Z}}\left(\rho\left(\frac{|n+1|}{k}\right) v_{n} \bar{v}_{n+1}+\rho\left(\frac{|n|}{k}\right) v_{n+1} \bar{v}_{n}\right) \\
& -\lambda \operatorname{Re} \sum_{n \in \mathbb{Z}}\left(\rho\left(\frac{|n+1|}{k}\right) v_{n} \bar{v}_{n+1}+\rho\left(\frac{|n|}{k}\right) v_{n+1} \bar{v}_{n}\right) \\
\geq & \alpha \operatorname{Im} \sum_{n \in \mathbb{Z}}\left(\rho\left(\frac{|n+1|}{k}\right) v_{n} \bar{v}_{n+1}+\rho\left(\frac{|n|}{k}\right) v_{n+1} \bar{v}_{n}\right) \\
= & \alpha \operatorname{Im} \sum_{n \in \mathbb{Z}}\left(\rho\left(\frac{|n+1|}{k}\right)-\rho\left(\frac{|n|}{k}\right)\right) v_{n} \bar{v}_{n+1},
\end{aligned}
$$

where we use the fact that

$$
\begin{aligned}
& \sum_{n \in \mathbb{Z}}\left(\rho\left(\frac{|n+1|}{k}\right)\left|v_{n+1}\right|^{2}+\rho\left(\frac{|n|}{k}\right)\left|v_{n}\right|^{2}\right) \\
& \geq \operatorname{Re} \sum_{n \in \mathbb{Z}}\left(\rho\left(\frac{|n+1|}{k}\right) v_{n} \bar{v}_{n+1}+\rho\left(\frac{|n|}{k}\right) v_{n+1} \bar{v}_{n}\right) .
\end{aligned}
$$


By the property of the cut-off function, we have

$$
\begin{aligned}
\left|\alpha \operatorname{Im} \sum_{j \in \mathbb{Z}}\left(\rho\left(\frac{|n+1|}{k}\right)-\rho\left(\frac{|n|}{k}\right)\right) v_{j} \bar{v}_{n+1}\right| & =\left|\alpha \operatorname{Im} \sum_{n \in \mathbb{Z}} \frac{\left|\rho^{\prime}\left(\xi_{n}\right)\right|}{k} v_{n} \bar{v}_{n+1}\right| \\
& \leq \frac{|\alpha| C_{0}}{k}\|v\|^{2}
\end{aligned}
$$

which yields

$$
-2 \operatorname{Re}((\lambda+\mathrm{i} \alpha) A v, x) \leq \frac{2|\alpha| C_{0}}{k}\|v\|^{2} .
$$

For the last term on the right-hand side of (3.23), we obtain

$$
2 e^{-\delta\left(\theta_{t} \omega\right)}(g(t), x) \leq \frac{\gamma}{2} \sum_{n \in \mathbb{Z}} \rho\left(\frac{|n|}{k}\right)\left|v_{n}\right|^{2}+\frac{2}{\gamma} e^{-2 \delta\left(\theta_{t} \omega\right)} \sum_{n \in \mathbb{Z}} \rho\left(\frac{|n|}{k}\right)\left|g_{n}(t)\right|^{2} .
$$

Then it follows from (3.23)-(3.25) that

$$
\begin{aligned}
\frac{d}{d t} \sum_{n \in \mathbb{Z}} \rho\left(\frac{|n|}{k}\right)\left|v_{n}\right|^{2} \leq & \frac{2|\alpha| C_{0}}{k}\|v\|^{2}-\left(\gamma-2 \delta\left(\theta_{t} \omega\right)\right) \sum_{n \in \mathbb{Z}} \rho\left(\frac{|n|}{k}\right)\left|v_{n}\right|^{2} \\
& +\frac{2}{\gamma} e^{-2 \delta\left(\theta_{t} \omega\right)} \sum_{n \in \mathbb{Z}} \rho\left(\frac{|n|}{k}\right)\left|g_{n}(t)\right|^{2}
\end{aligned}
$$

Given $t \in \mathbb{R}^{+}, \tau \in \mathbb{R}$, and $\omega \in \Omega$, it follows from (3.26) that

$$
\begin{aligned}
\sum_{n \in \mathbb{Z}} \rho\left(\frac{|n|}{k}\right)\left|v_{n}\left(\tau, \tau-t, \omega, v_{\tau-t}\right)\right|^{2} \\
\leq e^{-\gamma t+2 \int_{\tau-t}^{\tau} \delta\left(\theta_{r} \omega\right) d r} \sum_{j \in \mathbb{Z}} \rho\left(\frac{|n|}{k}\right)\left|v_{\tau-t, n}\right|^{2} \\
\quad+\frac{2|\alpha| C_{0}}{k} \int_{\tau-t}^{\tau} e^{\gamma(s-\tau)+2 \int_{s}^{\tau} \delta\left(\theta_{r} \omega\right) d r}\left\|\nu\left(s, \tau-t, \omega, v_{\tau-t}\right)\right\|^{2} d s \\
\quad+\frac{2}{\gamma} \int_{\tau-t}^{\tau} e^{\gamma(s-\tau)+2 \int_{s}^{\tau} \delta\left(\theta_{r} \omega\right) d r} e^{-2 \delta\left(\theta_{s} \omega\right)} \sum_{|n| \geq k}\left|g_{n}(s)\right|^{2} d s .
\end{aligned}
$$

We now replace $\omega$ in (3.27) by $\theta_{-\tau} \omega$ to yield

$$
\begin{aligned}
\sum_{n \in \mathbb{Z}} \rho\left(\frac{|n|}{k}\right)\left|v_{n}\left(\tau, \tau-t, \theta_{-\tau} \omega, v_{\tau-t}\right)\right|^{2} \\
\leq e^{-\gamma t+2 \int_{-t}^{0} \delta\left(\theta_{r} \omega\right) d r} \sum_{n \in \mathbb{Z}} \rho\left(\frac{|n|}{k}\right)\left|v_{\tau-t, n}\right|^{2} \\
\quad+\frac{2|\alpha| C_{0}}{k} \int_{-t}^{0} e^{\gamma s+2 \int_{s}^{0} \delta\left(\theta_{r} \omega\right) d r}\left\|v\left(s+\tau, \tau-t, \theta_{-\tau} \omega, v_{\tau-t}\right)\right\|^{2} d s \\
\quad+\frac{2}{\gamma} \int_{-\infty}^{0} e^{\gamma s+2 \int_{s}^{0} \delta\left(\theta_{r} \omega\right) d r} e^{-2 \delta\left(\theta_{s} \omega\right)} \sum_{|n| \geq k}\left|g_{n}(s+\tau)\right|^{2} d s .
\end{aligned}
$$


By the fact that $v_{\tau-t} \in D\left(\tau-t, \theta_{-t} \omega\right)$ and $D$ is tempered, we have

$$
\begin{aligned}
& \limsup _{t \rightarrow \infty} e^{-\gamma t+2 \int_{-t}^{0} \delta\left(\theta_{r} \omega\right) d r} \sum_{n \in \mathbb{Z}} \rho\left(\frac{|n|}{k}\right)\left|v_{\tau-t, n}\right|^{2} \\
& \quad \leq \limsup _{t \rightarrow \infty} e^{-\gamma t+2 \int_{-t}^{0} \delta\left(\theta_{r} \omega\right) d r}\left\|D\left(\tau-t, \theta_{-t} \omega\right)\right\|^{2}=0,
\end{aligned}
$$

which means that we can choose $T_{1}=T_{1}(\tau, \omega, D)>0$ such that, for all $t \geq T_{1}$,

$$
e^{-\gamma t+2 \int_{-t}^{0} \delta\left(\theta_{r} \omega\right) d r} \sum_{n \in \mathbb{Z}} \rho\left(\frac{|n|}{k}\right)\left|v_{\tau-t, n}\right|^{2} \leq \frac{\varepsilon}{3}
$$

By (3.12) and the properties of $z_{m}^{*}$, we know that

$$
\frac{2}{\gamma} \int_{-\infty}^{0} e^{\gamma s+2 \int_{s}^{0} \delta\left(\theta_{r} \omega\right) d r} e^{-2 \delta\left(\theta_{s} \omega\right)} \sum_{n \in \mathbb{Z}}\left|g_{n}(s+\tau)\right|^{2} d s<\infty
$$

and hence there is a $N_{1}=N_{1}(\tau, \omega, \varepsilon)>0$ such that, for all $k \geq N_{1}$,

$$
\frac{2}{\gamma} \int_{-\infty}^{0} e^{\gamma s+2 \int_{s}^{0} \delta\left(\theta_{r} \omega\right) d r} e^{-2 \delta\left(\theta_{s} \omega\right)} \sum_{|n| \geq k}\left|g_{n}(s+\tau)\right|^{2} d s \leq \frac{\varepsilon}{3}
$$

Furthermore, it follows from Lemma 3.2 that there exist $T_{2}=T_{2}(\tau, \omega, D, \varepsilon)>0$ and $N_{2}=$ $N_{2}(\tau, \omega, \varepsilon)>0$ such that, for all $t \geq T_{2}$ and $k \geq N_{2}$,

$$
\frac{2|\alpha| C_{0}}{k} \int_{\tau-t}^{\tau} e^{\gamma(s-\tau)+2 \int_{s}^{\tau} \delta\left(\theta_{r} \omega\right) d r}\left\|v\left(s, \tau-t, \omega, \nu_{\tau-t}\right)\right\|^{2} d s \leq \frac{\varepsilon}{3} .
$$

Denote by $\hat{N}(\tau, \omega, \varepsilon)=\max \left\{N_{1}, N_{2}\right\}$ and $T(\tau, \omega, D, \varepsilon)=\max \left\{T_{1}, T_{2}\right\}$, it follows from (3.28)(3.31) that for all $t \geq T(\tau, \omega, D, \varepsilon)$ and $k \geq \hat{N}(\tau, \omega, \varepsilon)$,

$$
\begin{aligned}
\sum_{|n| \geq 2 k}\left|v_{n}\left(\tau, \tau-t, \theta_{-\tau} \omega, v_{\tau-t}\right)\right|^{2} & \leq \sum_{n \in \mathbb{Z}} \rho\left(\frac{|n|}{k}\right)\left|v_{n}\left(\tau, \tau-t, \theta_{-\tau} \omega, v_{\tau-t}\right)\right|^{2} \\
& \leq \varepsilon
\end{aligned}
$$

which concludes the proof.

Based on the uniform estimates given by Lemmas 3.2 and 3.3, we are now ready to present the existence of $\mathcal{D}$-pullback attractors for $\Phi$.

Theorem 3.1 Suppose (3.12) and (3.13) hold. Then the continuous cocycle $\Phi$ associated with equation (3.3) and (3.4) has a unique $\mathcal{D}$-pullback attractor $\mathcal{A}=\{\mathcal{A}(\tau, \omega): \tau \in \mathbb{R}, \omega \in$ $\Omega\} \in \mathcal{D}$ in $\ell^{2}$.

Proof Notice that, for each $\tau \in \mathbb{R}, \omega \in \Omega$,

$$
u\left(\tau, \tau-t, \theta_{-\tau} \omega, u_{\tau-t}\right)=e^{\delta(\omega)} v\left(\tau, \tau-t, \theta_{-\tau} \omega, v_{\tau-t}\right),
$$


where $u_{\tau-t}=e^{-\delta\left(\theta_{-t} \omega\right)} v_{\tau-t}$. Suppose $D=\{D(\tau, \omega): \tau \in \mathbb{R}, \omega \in \Omega\}$ is a family of nonempty subsets of $\ell^{2}$. Based on $D$, define a family $\hat{D}$ by

$$
\hat{D}(\tau, \omega)=\left\{\varphi \in \ell^{2}:\|\varphi\|^{2} \leq e^{-\delta\left(\theta_{-\tau} \omega\right)}\|D(\tau, \omega)\|^{2}, \tau \in \mathbb{R}, \omega \in \Omega\right\} .
$$

If $D$ is tempered, then one can check that $\hat{D}$ given by (3.34) is also tempered. In addition, if $u_{\tau-t} \in D\left(\tau-t, \theta_{-t} \omega\right)$, then we have

$$
v_{\tau-t}=e^{-\delta\left(\theta_{-t} \omega\right)} u_{\tau-t} \in \hat{D}\left(\tau-t, \theta_{-t} \omega\right)
$$

For every $\tau \in \mathbb{R}$ and $\omega \in \Omega$, denote $K(\tau, \omega)$ by

$$
K(\tau, \omega)=\left\{\varphi \in \ell^{2}:\|\varphi\|^{2} \leq \frac{4}{\gamma} e^{2 \delta(\omega)} \int_{-\infty}^{0} e^{\gamma s+2 \int_{s}^{0} \delta\left(\theta_{r} \omega\right) d r} e^{-2 \delta\left(\theta_{s} \omega\right)}\|g(s+\tau)\|^{2} d s\right\} .
$$

Since $\hat{D} \in \mathcal{D}$, by Lemma 3.2, we find that there exists $T=(\tau, \omega, D)>0$ such that, for all $t \geq T$ and $v_{\tau-t} \in \hat{D}\left(\tau-t, \theta_{-t} \omega\right)$,

$$
\begin{aligned}
\left\|u\left(\tau, \tau-t, \theta_{-t} \omega, u_{\tau-t}\right)\right\|^{2} & \leq e^{2 \delta(\omega)}\left\|v\left(\tau, \tau-t, \theta_{-t} \omega, v_{\tau-t}\right)\right\|^{2} \\
& \leq \frac{4}{\gamma} e^{2 \delta(\omega)} \int_{-\infty}^{0} e^{\gamma s+2 \int_{s}^{0} \delta\left(\theta_{r} \omega\right) d r} e^{-2 \delta\left(\theta_{s} \omega\right)}\|g(s+\tau)\|^{2} d s,
\end{aligned}
$$

which means that, for all $t \geq T$,

$$
\Phi\left(t, \tau-t, \theta_{-t} \omega, D\left(\tau-t, \theta_{-t} \omega\right)\right) \subseteq K(\tau, \omega)
$$

This shows that $K$ pullback-attracts all elements in $\mathcal{D}$. We now verify that $K$ given by (3.35) is tempered. Let $\xi$ be an arbitrary positive number. Then for each $\tau \in \mathbb{R}$ and $\omega \in \Omega$, we have by (3.35)

$$
\begin{aligned}
e^{\xi t} & \left\|K\left(\tau+t, \theta_{t} \omega\right)\right\|^{2} \\
& \leq \frac{4}{\gamma} e^{\xi t} e^{2 \delta\left(\theta_{t} \omega\right)} \int_{-\infty}^{0} e^{\gamma s+2 \int_{s}^{0} \delta\left(\theta_{r+t} \omega\right) d r} e^{-2 \delta\left(\theta_{s+t} \omega\right)}\|g(s+\tau+t)\|^{2} d s \\
& =\frac{4}{\gamma} e^{\xi t} e^{2 \delta\left(\theta_{t} \omega\right)} \int_{-\infty}^{0} e^{\gamma s+2 \int_{s+t}^{t} \delta\left(\theta_{r} \omega\right) d r} e^{-2 \delta\left(\theta_{s+t} \omega\right)}\|g(s+\tau+t)\|^{2} d s .
\end{aligned}
$$

Let $0<\varepsilon<\frac{1}{8} \min \{\xi, \gamma\}$. By the properties of $z_{m}^{*}(\omega)$, we find that

$$
\left|\int_{0}^{t} \delta\left(\theta_{s} \omega\right) d s\right| \leq \varepsilon|t|, \quad\left|\delta\left(\theta_{t} \omega\right)\right| \leq \varepsilon|t|
$$

for $|t|$ large enough. Therefore, (3.38) implies that

$$
\begin{aligned}
& \lim _{t \rightarrow-\infty} e^{\xi t}\left\|K\left(\tau+t, \theta_{t} \omega\right)\right\|^{2} \\
& \quad \leq \frac{4}{\gamma} \lim _{t \rightarrow-\infty} \int_{-\infty}^{0} e^{\xi t+2 \varepsilon|t|+\gamma s+4 \varepsilon|s+t|+2 \varepsilon|t|}\|g(s+\tau+t)\|^{2} d s \\
& \quad \leq \frac{4}{\gamma} \lim _{t \rightarrow-\infty} e^{(\xi-8 \varepsilon) t} \int_{-\infty}^{0} e^{(\gamma-4 \varepsilon) s}\|g(s+\tau+t)\|^{2} d s .
\end{aligned}
$$


Then it follows from (3.13) and (3.39) that

$$
\begin{aligned}
& \lim _{t \rightarrow-\infty} e^{\xi t}\left\|K\left(\tau+t, \theta_{t} \omega\right)\right\|^{2} \\
& \leq \frac{4}{\gamma} \lim _{t \rightarrow-\infty} e^{(\xi-8 \varepsilon) t} \int_{-\infty}^{0} e^{\frac{\gamma}{2} s}\|g(s+\tau+t)\|^{2} d s=0 .
\end{aligned}
$$

Therefore we find from (3.40) that $K$ is tempered in $\ell^{2}$. Moreover, we can check that $K$ is measurable. Thus, $K \in \mathcal{D}$ is a closed measurable $\mathcal{D}$-pullback absorbing set for $\Phi$.

By a similar argument, from Lemma 3.3, we can derive the following estimates on the tails of solutions of (3.3)-(3.4). For every $\tau \in \mathbb{R}, \omega \in \Omega, D=\{D(\tau, \omega): \tau \in \mathbb{R}, \omega \in \Omega\} \in \mathcal{D}$ and $\varepsilon>0$, there exist $T=T(\tau, \omega, D, \varepsilon)>0$ and $\hat{N}=\hat{N}(\tau, \omega, \varepsilon)>0$ such that, for all $t \geq T$ and $\lambda \geq \lambda_{0}$, the solution of problem (3.1)-(3.2) satisfies

$$
\sum_{|i| \geq \hat{N}}\left|u_{i}\left(\tau, \tau-t, \theta_{-\tau} \omega, u_{\tau-t}\right)\right|^{2} \leq \varepsilon
$$

where $u_{\tau-t} \in D\left(\tau-t, \theta_{-t} \omega\right)$. This implies that $\Phi$ is asymptotically null in $\ell^{2}$. Thus Theorem 3.1 follows from Theorem 3.6 in [29] immediately.

We now consider the periodicity of the attractor $\mathcal{A}$. Suppose that $g(t)$ is $T$-periodic function with respect to $t$ and $g \in L^{2}\left((0, T), \ell^{2}\right)$. Then by (3.35) we find that the $\mathcal{D}$-pullback absorbing set is $T$-periodic. Furthermore, in this case, the cocycle $\Phi$ associated with problem (3.3) and (3.4) is also $T$-periodic. Thus from Proposition 2.1, the periodicity of the attractor $\mathcal{A}$ follows.

Theorem 3.2 Let (3.12) and (3.13) hold. Suppose that $g(t)$ is T-periodic function and $g \in$ $L^{2}\left((0, T), \ell^{2}\right)$ for $T>0$. Then the continuous cocycle $\Phi$ associated with (3.3) and (3.4) has a unique $T$-periodic $\mathcal{D}$-pullback attractor $\mathcal{A} \in \mathcal{D}$ in $\ell^{2}$.

\section{Competing interests}

The authors declare that they have no competing interests.

\section{Authors' contributions}

The authors have contributed to this work on an equal basis. All authors read and approved the final manuscript.

\section{Author details}

${ }^{1}$ College of Mathematics and Software Science, Sichuan Normal University, Chengdu, 610068, China. ${ }^{2}$ School of Science, Xihua University, Chengdu, 610039, China. ${ }^{3}$ Colloge of Mathematics, Sichuan University, Chengdu, 610064, China.

\section{Acknowledgements}

The work is supported by National Natural Science Foundation of China (11331007, 11201320).

Received: 27 March 2015 Accepted: 13 July 2015 Published online: 30 July 2015

\footnotetext{
References

1. Chow, SN, Mallet-Paret, J, Shen, W: Traveling waves in lattice dynamical systems. J. Differ. Equ. 149, 248-291 (1998)

2. Bates, PW, Chmaj, A: A discrete convolution model for phase transitions. Arch. Ration. Mech. Anal. 150, 281-305 (1999)

3. Zinner, B: Existence of traveling wavefront solutions for the discrete Nagumo equation. J. Differ. Equ. 96, 1-27 (1992)

4. Chow, SN, Paret, JM: Pattern formation and spatial chaos in lattice dynamical systems. IEEE Trans. Circuits Syst. 42, 746-751 (1995)

5. Chow, SN, Shen, WX: Dynamics in a discrete Nagumo equation: spatial topological chaos. SIAM J. Appl. Math. 55, 1764-1781 (1995)

6. Shen, WX: Lifted lattices, hyperbolic structures, and topological disorders in coupled map lattices. SIAM J. Appl. Math. 56, 1379-1399 (1996)

7. Bates, PW, Lu, KN, Wang, BX: Attractor for lattice dynamical systems. Int. J. Bifurc. Chaos 1, 143-153 (2001)
} 
8. Wang, BX: Dynamics of systems on infinite lattices. J. Differ. Equ. 221, 224-245 (2006)

9. Wang, BX: Asymptotic behavior of non-autonomous lattice systems. J. Math. Anal. Appl. 331, 121-136 (2007)

10. Zhou, SF: Attractors for lattice systems corresponding to evolution equations. Nonlinearity 15, 1079-1095 (2002)

11. Zhou, SF: Attractors for second order lattice dynamical systems. J. Differ. Equ. 179, 605-624 (2002)

12. Zhou, SF: Attractors for first order dissipative lattice dynamical systems. Physica D 178, 51-61 (2003)

13. Karachalios, NI, Yannacopoulos, AN: The existence of a global attractor for the discrete nonlinear Schrödinger equation, II. Compactness without tail estimates in $\mathbb{Z}^{N}, N \geq 1$, lattices. Proc. R. Soc. Edinb. 137, $63-76$ (2007)

14. Karachalios, NI, Nistazakis, HE, Yannacopoulos, AN: Remarks on the asymptotic behavior of solutions of complex discrete Ginzburg-Landau equations. Discrete Contin. Dyn. Syst., Ser. A suppl., 476-486 (2005)

15. Zhao, CD, Zhou, SF: Limit behavior of global attractors for the complex Ginzburg-Landau equation on infinite lattices. Appl. Math. Lett. 21, 628-635 (2008)

16. Staliunas, $\mathrm{K}:$ Spatial and temporal noise spectra of spatially extended systems with order-disorder phase transitions. Int. J. Bifurc. Chaos 11, 2845-2852 (2001)

17. Bates, PW, Lisei, H, Lu, KN: Attractors for stochastic lattice dynamical systems. Stoch. Dyn. 6, 1-21 (2006)

18. Caraballo, T, Lu, KN: Attractors for stochastic lattice dynamical systems with a multiplicative noise. Front. Math. China 3, 317-335 (2008)

19. Wang, $\mathrm{XH}, \mathrm{Li}, \mathrm{SY}, \mathrm{Xu}, \mathrm{DY}$ : Random attractors for second-order stochastic lattice dynamical systems. Nonlinear Anal. 72 , 483-494 (2012)

20. Huang, JH: The random attractor of stochastic FitzHugh-Nagumo equations in an infinite lattice with white noises. Physica D 233, 83-94 (2007)

21. Zhou, SF, Wei, L: A random attractor for a stochastic second order lattice system with random coupled coefficients. J. Math. Anal. Appl. 395, 42-55 (2012)

22. Zhou, SF, Wang, ZJ: Random attractors for stochastic retarded lattice systems. J. Differ. Equ. Appl. 19, 1523-1543 (2013)

23. Gu, AH, Ai, W: Random attractor for stochastic lattice dynamical systems with $\alpha$-stable Lévy noises. Commun. Nonlinear Sci. Numer. Simul. 19, 1433-1441 (2014)

24. $\mathrm{Gu}, \mathrm{AH}, \mathrm{Li}, \mathrm{YR}, \mathrm{Li}$, J: Random attractors on lattice of stochastic FitzHugh-Nagumo systems driven by $\alpha$-stable Lévy noises. Int. J. Bifurc. Chaos 24, Article ID 1450123 (2014)

25. LV, Y, Sun, JH: Asymptotic behavior of stochastic discrete complex Ginzburg-Landau equations. Physica D 221, 157-169 (2006)

26. Wang, BX: Sufficient and necessary criteria for existence of pullback attractors for non-compact random dynamical systems. J. Differ. Equ. 253, 1544-1583 (2012)

27. Wang, BX: Existence and upper semicontinuity of attractors for stochastic equations with deterministic non-autonomous terms. Stoch. Dyn. 14, 1-31 (2014)

28. Adili, A, Wang, BX: Random attractors for stochastic FitzHugh-Nagumo systems driven by deterministic nonautonomous forcing. Discrete Contin. Dyn. Syst., Ser. B 18, 643-666 (2013)

29. Bates, PW, Lu, KN, Wang, BX: Attractors of non-autonomous stochastic lattice systems in weighted spaces. Physica D 289, $32-50(2014)$

30. Li, YR, Cui, HY: Pullback attractor for nonautonomous Ginzburg-Landau equation with additive noise. Abstr. Appl. Anal. 2014, Article ID 921750 (2014)

31. Crauel, H, Flandoli, F: Attractor for random dynamical systems. Probab. Theory Relat. Fields 100, 365-393 (1994)

32. Flandoli, F, Schmalfuss, B: Random attractors for the 3D stochastic Navier-Stokes equation with multiplicative noise. Stoch. Stoch. Rep. 59, 21-45 (1996)

33. Schmalfuss, B: Backward cocycle and attractors of stochastic differential equations. In: Reitmann, V, Riedrich, T, Koksch, N (eds.) International Seminar on Applied Mathematics-Nnonlinear Dynamics: Attractor Approximation and Global Behavior, pp. 185-192. Technische Universität, Berlin (1992)

34. Arnold, L: Random Dynamical System. Springer, Berlin (1998)

35. Caraballo, T, Garrido-Atienza, MJ, Schmalfuss, B: Exponential stability of stationary solutions for semilinear stochastic evolution equations with delays. Discrete Contin. Dyn. Syst. 18, 271-293 (2007)

36. Caraballo, T, Kloeden, PE, Schmalfuss, B: Exponentially stable stationary solutions for stochastic evolutions equations and their perturbations. Appl. Math. Optim. 50, 183-207 (2004)

37. Chepyzhov, VV, Vishik, Ml: Attractors for Equations of Mathematical Physics, vol. 49. Am. Math. Soc., Providence (2002) 